\title{
Photoelastic Analysis of Edge Residual Stresses in Glass by Automated "Test Fringes" Methods
}

\author{
A. Ajovalasit • G. Petrucci • M. Scafidi
}

Received: 15 April 2011 / Accepted: 14 September 2011

(C) Society for Experimental Mechanics 2011

\begin{abstract}
Since the glass is a birefringent material, the analysis of residual stress in glass is usually carried out by means of photoelastic methods. This paper considers the automation of the "test fringes" method which is based on the use of a Babinet compensator or of a beam subjected to bending. In particular, two automated methods are proposed: the first one is based on the use of the centre fringe method in monochromatic light and the second one is based on the use of $R G B$ photoelasticity in white light. The proposed methods have been applied to the analysis of membranal residual stresses in some tempered glasses, showing that they can effectively replace manual methods of photoelastic analysis of residual stresses in glass.
\end{abstract}

Keywords Glass · Residual stresses · Digital photoelasticity $\cdot$ Image processing

\section{Introduction}

It is known that photoelasticity can be used for residual stress analysis of glass [1-3]. The development of digital photoelasticity [4, 5] allows the user to automate the analysis of residual stress in glass as shown in Ref. [6] in the case of the phase shifting method.

\footnotetext{
A. Ajovalasit $(\varangle) \cdot$ G. Petrucci $\cdot$ M. Scafidi

University of Palermo (Italy),

Viale delle Scienze,

90128 Palermo, Italy

e-mail: a.ajovalasit@gmail.com

G. Petrucci

e-mail: giovanni.petrucci@unipa.it

M. Scafidi

e-mail: michele.scafidi@unipa.it
}

Residual stresses in a glass specimen can be easily detected by inserting a Babinet compensator or even a simple bent beam in front of or behind the glass. The resulting fringes, which are sometimes called test fringes [1], effectively reveal the presence of residual stresses. For example, Figure 1 shows the isochromatic fringes in the glass specimen alone $(\mathrm{a}, \mathrm{b})$, in the bent beam alone (c) and finally the resulting fringes in the glass specimen superimposed to the bent beam (d). The shape of the resulting fringes clearly reveals the residual stress. As can be seen in Fig. 1, the vertical reference fringes are modulated by the residual stresses in the glass specimen. The deviation of the fringes immediately puts in evidence the presence of residual stresses, although in a qualitative way. Another effect of the reference fringes is that, in general, the retardation in the glass specimen increases. This is a favourable effect because it increases the retardation in zones, near the 0 order fringes, where it is too low.

The quantitative analysis can be performed using the laborious manual technique based on the use of compensation methods.

In this paper the automation of the test fringes method is proposed by using two methods:

1. the Centre Fringe Method (CFM) in monochromatic light [4];

2. the RGB photoelasticity in white light [7-9].

The paper also shows the application of the proposed methods to the analysis of membranal residual stresses in some tempered glasses, in particular a household shelf and the rear window of a car.

The glass specimen and the bent beam must be placed in such a way that the principal stresses in both elements are properly aligned, like in the cases of the manual method 


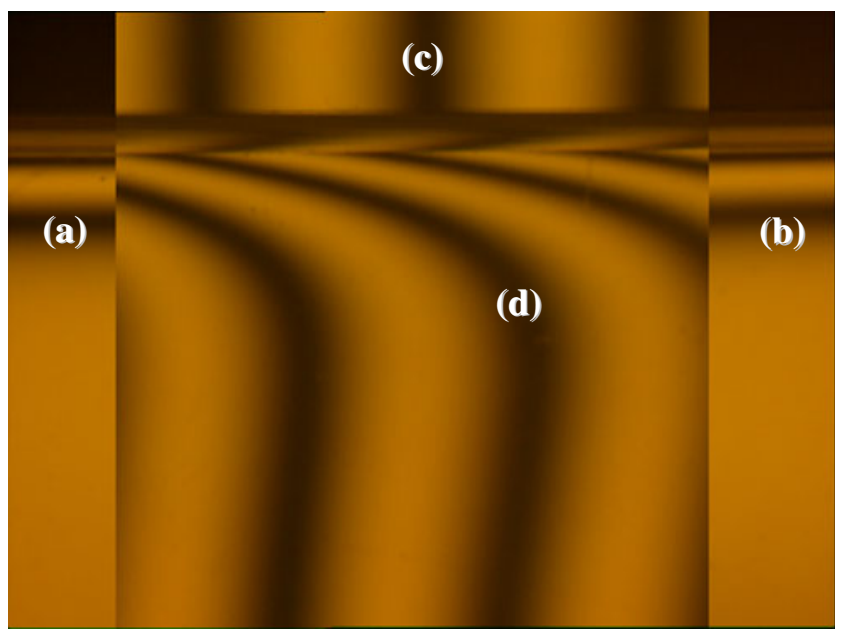

Fig. 1 (a), (b) isochromatic fringes in the glass specimen alone, (c) carrier fringes in the bent beam alone, (d) resulting isochromatic fringes (test fringes) due to the superposition of the glass specimen and of the bent beam

based on the Babinet compensator and of the Fourier transform method of fringe analysis [10].

\section{Theory}

In the test fringes method, a system of carrier fringes (see Fig. 2) is superimposed on the glass specimen placed in a circular polariscope. Such a system of carrier fringes is obtained by using a Babinet compensator or a bent beam, which is a specimen subjected to bending. In the present work, the second solution has been used.

\section{Use of Monochromatic Light}

In the case of monochromatic light source, the intensity emerging from the dark field circular polariscope shown in Fig. 2 is given by the relationship valid for the Fourier transform method of fringe analysis [10]. Without consid- ering the disturbances due to background intensity and noise, this relationship can be written as follows:

$I=\frac{I_{0}}{2}\left[1-\sin ^{2}\left(\alpha-\alpha_{c}\right) \cos 2 \pi\left(\delta^{c}-\delta\right)-\cos ^{2}\left(\alpha-\alpha_{c}\right) \cos 2 \pi\left(\delta^{c}+\delta\right)\right]$

where $I_{0}$ is the maximum light intensity, $\alpha_{c}$ and $\alpha$ are the angles that define the directions of the maximum principal stress respectively in the carrier and in the glass (Fig. 2(b)), $\delta^{\mathrm{c}}$ is the retardation (known) in the carrier and $\delta$ is the retardation in the glass specimen. The retardation $\delta$ is related to the membranal residual stresses $\sigma_{1}, \sigma_{2}\left(\sigma_{1} \geq \sigma_{2}\right)$ existing in the glass by the relationship

$\delta=\frac{C_{\lambda} d}{\lambda}\left(\sigma_{1}-\sigma_{2}\right)$

where $C_{\lambda}$ is the stress optical coefficient, $d$ is the glass thickness and $\lambda$ is the wavelength of the light source.

Equation (1) shows that in photoelasticity, unlike to what happens in other optical methods such as holography and moiré, the light intensity depends on two parameters related to the stress field, i.e. the retardation $\delta$ and the isoclinic angle $\alpha$. Only in special conditions:

$a-a_{c}=0^{\circ}$ or $a-a_{c}= \pm 90^{\circ}$

the light intensity depends only on the retardation. In these cases equation (1) provides:

$I=\frac{I_{0}}{2}\left[1-\cos 2 \pi\left(\delta^{c} \pm \delta\right)\right]$

where the upper sign applies to $\alpha-\alpha_{c}=0^{\circ}$ and the lower to $\alpha-\alpha_{c}= \pm 90^{\circ}$. In these cases equation (4) gives the total retardation as follows:

for $a-a_{c}=0^{\circ}: \delta^{\text {tot }}=\delta^{c}+\delta$

for $a-a_{c}= \pm 90^{\circ}$ and $\delta^{c}>\delta: \delta^{\text {tot }}=\delta^{c}-\delta$
Fig. 2 (a) polariscope, (b) orientation of the optical elements. (c: carrier, P,A: polarizers, $\mathrm{R}_{\mathrm{p}}, \mathrm{R}_{\mathrm{a}}$ : quarter wave plates, $\sigma_{1}, \sigma_{2}$ : glass principal stresses, $\sigma_{1 \mathrm{c}}, \sigma_{2 \mathrm{c}}$ : carrier principal stresses, $\alpha, \alpha_{c}$ : orientation of principal stresses in the glass and in the carrier, TVC: CCD camera, PC: personal computer)

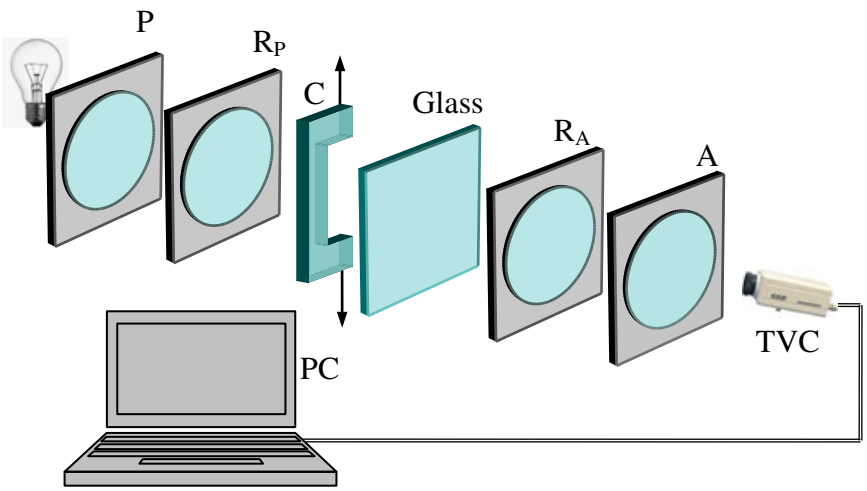

(a)

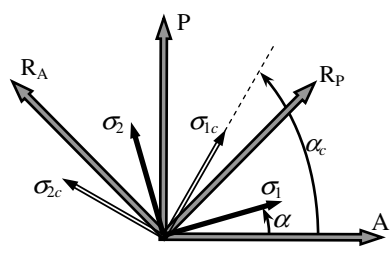

(b) 
for $a-a_{c}= \pm 90^{\circ}$ and $\delta^{c}<\delta: \delta^{\text {tot }}=-\left(\delta^{c}-\delta\right)$

The equations (5)-(7) can be written as

$\delta^{t o t}=\left|\delta^{c} \pm \delta\right|$

where the upper sign applies to $\alpha-\alpha_{c}=0^{\circ}$ and the lower to $\alpha-\alpha_{c}= \pm 90^{\circ}$.

From equations (5)-(8) it follows:

$\delta=\left|\delta^{t o t}-\delta^{c}\right|$ for $\left\{\begin{array}{l}\alpha-\alpha_{c}=0^{\circ}, \text { or } \\ \alpha-\alpha_{c}= \pm 90^{\circ}, \quad \delta^{c}>\delta .\end{array}\right.$

$\delta=\delta^{t o t}+\delta^{c}$ for $\alpha-\alpha_{c}= \pm 90^{\circ}, \quad \delta^{c}<\delta$

Once the retardation $\delta$ in the glass is known, the difference of principal stresses is obtained by using equation (2). Especially at the edges (usually compressed, that is $\sigma_{1}=0$ ), the value of the boundary stress is:

$\sigma_{2}=-\frac{\lambda}{C d} \delta$

\section{Use of the Centre Fringe Method (CFM)}

In the case of a carrier with retardation linearly varying in $x$ direction (vertical carrier fringes) and glass with constant retardation in $x$ direction (horizontal glass fringes), the total retardation (Fig. 3) given by equation (8) can be rewritten as follows

$\delta^{t o t}(x, y)=\left|\delta^{c}(x) \pm \delta(y)\right|$

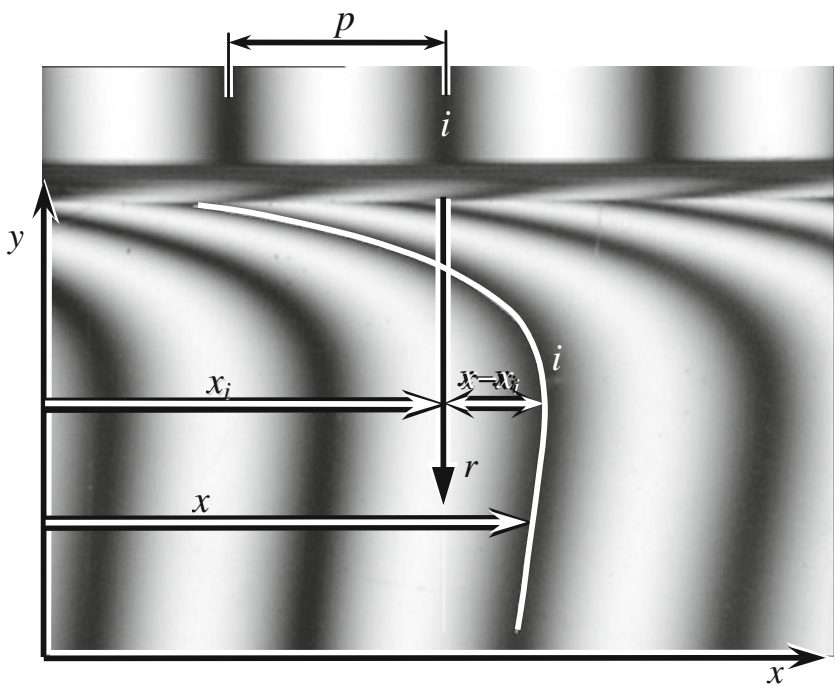

Fig. 3 Isochromatic fringes in glass superimposed on the carrier (down) and in the carrier alone (up) in monochromatic light
The retardation in the compensator can be written as:

$\delta^{c}(x)=\delta_{i}^{c} \pm \frac{x-x_{i}}{p}$

where $\delta_{i}^{c}$ is the retardation at the carrier fringe of abscissa $x_{i}, p$ is the pitch of the carrier fringes and the sign should be considered positive if the carrier retardation is increasing with the abscissa $x$ and vice versa. Taking account of (13), equation (12) can be written as:

$\delta^{t o t}(x, y)=\left|\delta_{i}^{c} \pm \frac{x-x_{i}}{p} \pm \delta(y)\right|$

Considering the points at which $\delta^{t o t}=\delta_{i}^{c}$ on the glass plate superimposed to the carrier, i.e. the centres of the fringe having retardation $\delta_{i}^{c}=i$ and abscissa $x$ (Fig. 3), equation (14) becomes

$\delta^{t o t}(x, y)=\delta_{i}^{c}=\left|\delta_{i}^{c} \pm \frac{x-x_{i}}{p} \pm \delta(y)\right|$

which lead to the following relationship

$\delta(y)=\frac{\left|x-x_{i}\right|}{p}$

Equation (16) shows that the retardation $\delta(y)$ can be evaluated by selecting a fringe of ascissa $x_{i}$ and retardation $\delta_{i}^{c}=i$ on the carrier and determining the $x$ coordinates of the centres of the fringe of the same order $i$ on the glass.

According to equation (16), and with reference to Fig. 3, the procedure for the determination of $\delta(y)$ is based on the following steps:

1. selection of a fringe of order $i$ (retardation $\delta_{i}^{c}$ and abscissa $x_{\mathrm{i}}$ ) on the carrier,

2 . selection of the fringe of the same order $i$ (retardation $\delta^{t o t}=\delta_{i}^{c}$ ) on the glass plate,

3. determination of the $x$ coordinates of the centre of the fringe by a centre fringe extraction procedure (see Appendix),

4. evaluation of the retardation $\delta(y)$ on the glass plate by equation (16)

\section{Use of White Light}

As previously said, after the superposition of the reference fringes, the retardation is, in general, increased. This effect may enhance the accuracy of methods based on the use of white light, which can be prone to large errors for low levels of retardation, like in the case of the RGB method [7].

Equation (4) can also be written as follows:

$I=I_{0} \sin ^{2} \pi\left(\delta^{c} \pm \delta\right)$ 


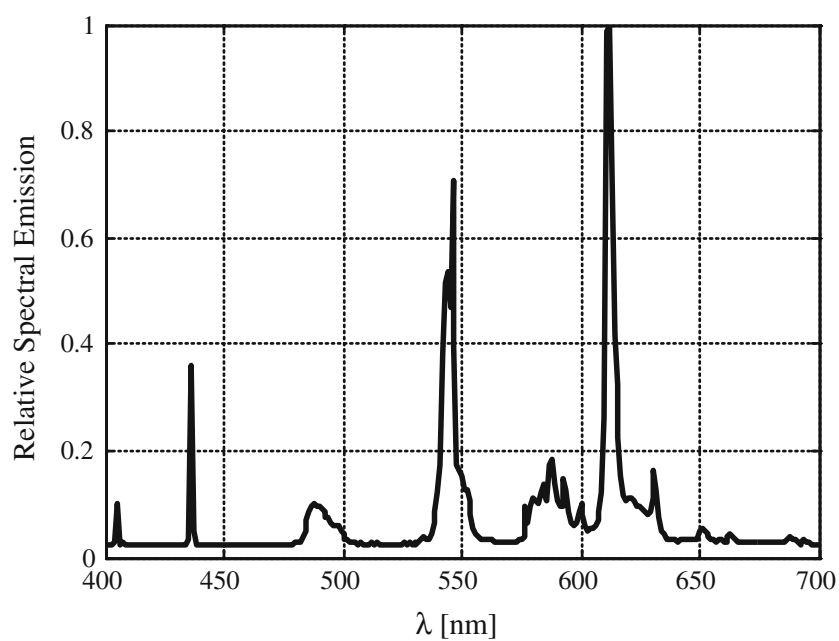

Fig. 4 Spectrum of emission of the fluorescent lamps with discrete spectrum

In white light, ignoring for simplicity the error due to the quarter-wave plates [9], equation (17) becomes:

$$
\begin{aligned}
I_{w j}= & \frac{1}{\lambda_{j 2}-\lambda_{j 1}} \int_{\lambda_{j 1}}^{\lambda_{j 2}} I_{0}(\lambda) T(\lambda) T^{c}(\lambda) F_{j}(\lambda) \sin ^{2} \pi \frac{\lambda_{0}}{\lambda} \\
& \times\left(\delta_{0}^{c} \frac{C_{\lambda}^{c}}{C_{0}^{c}} \pm \delta_{0} \frac{C_{\lambda}}{C_{0}}\right) d \lambda,(j=R, G, B)
\end{aligned}
$$

where $T(\lambda)$ and $T^{c}(\lambda)$ denote the transmittance of the glass and of the carrier, $\delta_{0}$ and $\delta_{0}{ }^{c}$ denote the retardation in the glass and in the carrier at the reference wavelength $\lambda_{0}$, $C_{\lambda} / C_{0}$ and $C_{\lambda}^{c} / C_{0}^{c}$ denote the dispersion of birefringence

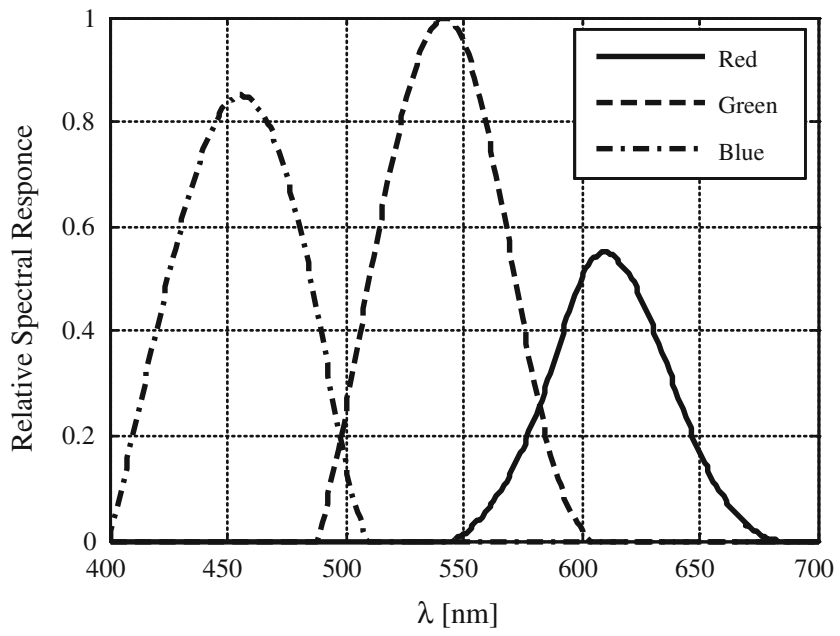

Fig. 5 Approximated spectral distributions of the filters R, G and B of the used camera

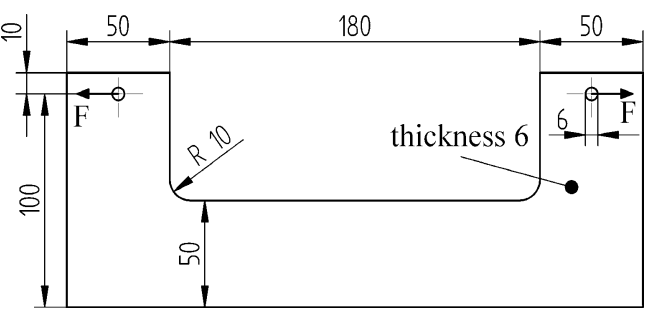

Fig. 6 Polycarbonate specimen (dimensions in $\mathrm{mm}$ ) used both as carrier and as a calibration beam for the RGB method

$[11,12]$ in the glass and in the carrier. If the dispersions of the two materials are equal, it follows that:

$\frac{C_{\lambda}^{c}}{C_{0}^{c}}=\frac{C_{\lambda}}{C_{0}}$

and consequently equation (18) becomes:

$I_{w j}=\frac{1}{\lambda_{j 2}-\lambda_{j 1}} \int_{\lambda_{j 1}}^{\lambda_{j 2}} I_{0}(\lambda) T(\lambda) T^{c}(\lambda) F_{j}(\lambda) \sin ^{2}\left[\pi \frac{\lambda_{0}}{\lambda} \frac{C_{\lambda}}{C_{0}}\left(\delta_{0}^{c} \pm \delta_{0}\right)\right](20)$ $d \lambda,(j=R, G, B)$

According to equation (20), the search procedure of RGB photoelasticity yields the total retardation

$\delta_{0}^{\text {tot }}=\left|\delta_{0}^{c} \pm \delta_{0}\right|$

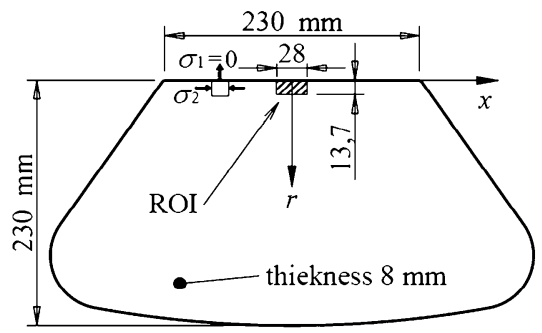

(a)

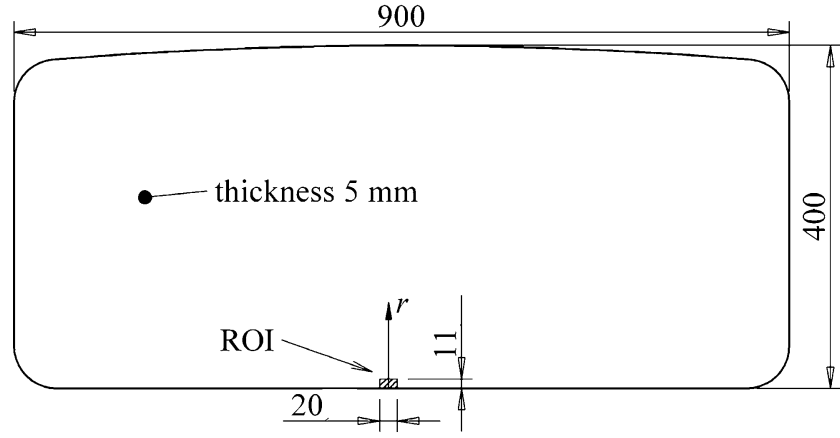

(b)

Fig. 7 Tempered glasses used in the experiments (dimensions in $\mathrm{mm}$ ): (a) household shelf, (b) rear window of car 
Fig. 8 Shelf: isochromatic

fringes in the carrier alone in white (a) and monochromatic (b) light and (c) at the ROI of the glass superimposed to the carrier

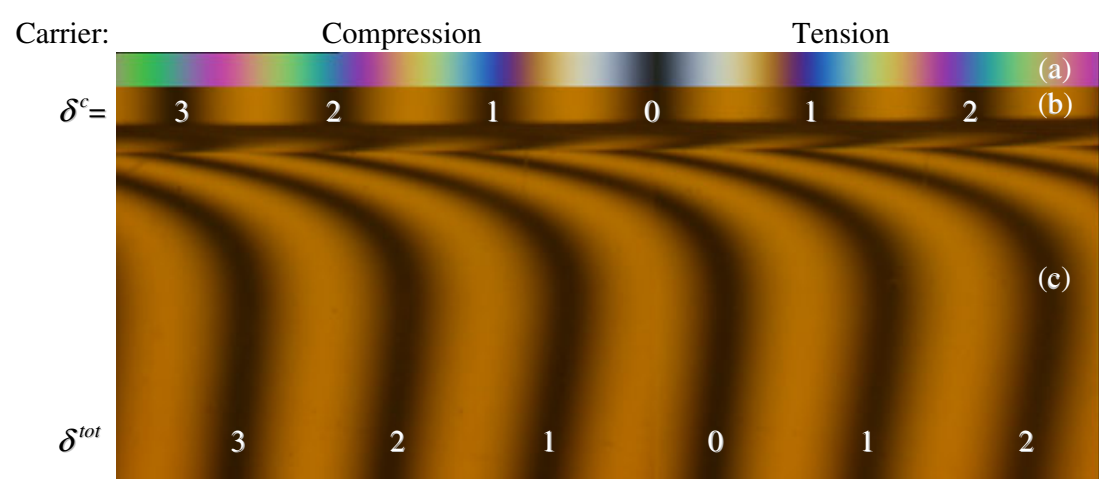

In the carrier alone, equation (20) provides:

$$
\begin{aligned}
I_{w j}^{c}= & \frac{1}{\lambda_{j 2}-\lambda_{j 1}} \int_{\lambda_{j 1}}^{\lambda_{j 2}} I_{0}(\lambda) T^{c}(\lambda) F_{j}(\lambda) \sin ^{2} \pi \frac{\lambda_{0}}{\lambda} \\
& \times \frac{C_{\lambda}}{C_{0}} \delta_{0}^{c} d \lambda, \quad(j=R, G, B)
\end{aligned}
$$

In this case RGB photoelasticity yields the retardation $\delta_{0}{ }^{c}$ in the carrier.

Note that the calibration step of RGB photoelasticity and the determination of the retardation in the carrier alone should be both done on the carrier superimposed to a sample of the same glass to be analysed, but free from residual stresses. In this research, the calibration has been performed in the carrier alone; this is equivalent to admit that it is:

$$
T(\lambda) T_{c}(\lambda)=T_{c}(\lambda)
$$

namely, that the transmittance of the glass is:

$$
T(\lambda)=1
$$

Once $\delta_{0}^{\text {tot }}$ and $\delta_{0}^{c}$ are determined, the retardation $\delta_{0}$ in the glass plate is calculated using the same relationships obtained in monochromatic light [see equations (9)-(10)], that are written as follows:

for $\alpha-\alpha_{c}=0^{\circ}$ and for $\alpha-\alpha_{c}= \pm 90^{\circ}$ with $\delta^{c}>\delta$, equation (9) becomes

$\delta_{0}=\left|\delta_{0}^{t o t}-\delta_{0}^{c}\right|$

whereas for $\alpha-\alpha_{c}= \pm 90^{\circ}$ with $\delta^{c}<\delta$, equation (10) becomes

$\delta_{0}=\delta_{0}^{t o t}+\delta_{0}^{c}$
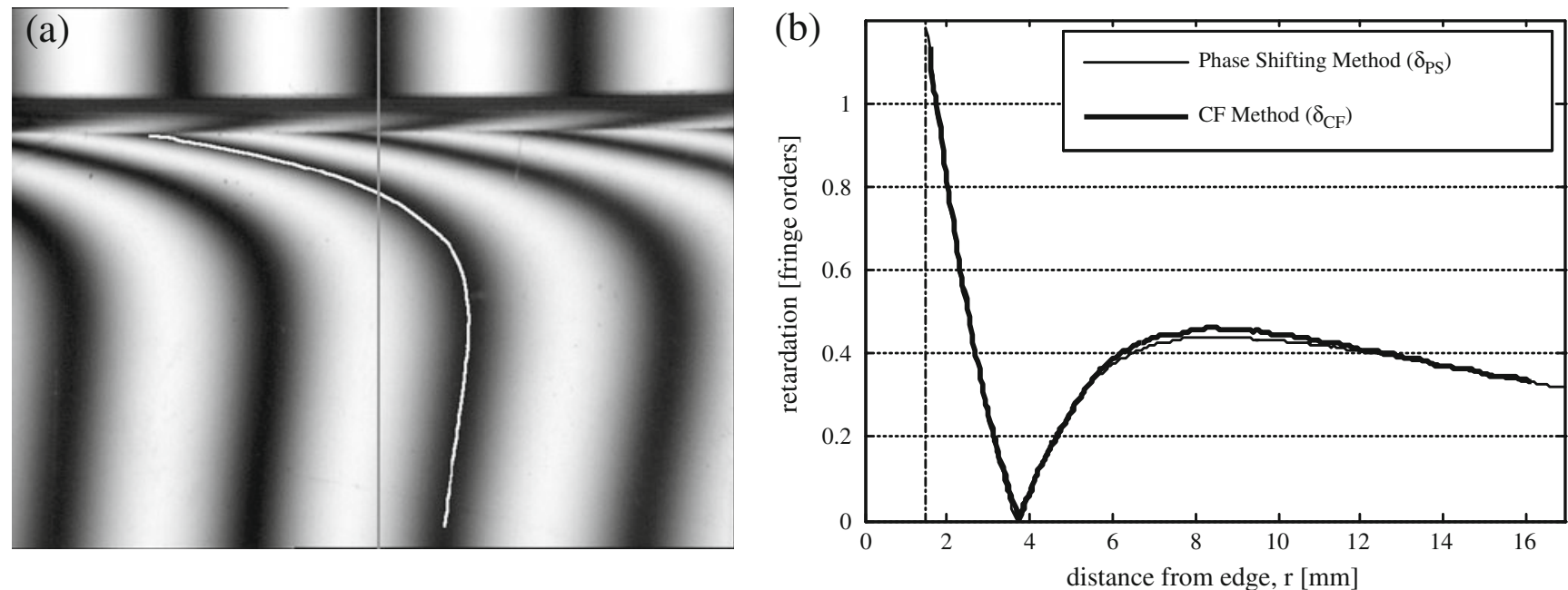

Fig. 9 Shelf: (a) isochromatic fringes in the ROI (the dark fringes used for the CFM method are marked-up), (b) Retardation $\delta$ along the direction $r$ normal to the contour of the glass determine by the CF method 


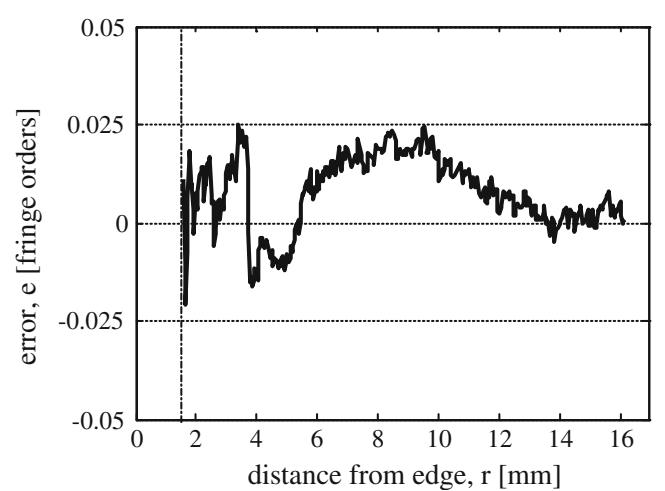

Fig. 10 Shelf: error $e=\delta_{C F}-\delta_{P S}$ between the results obtained by the $\mathrm{CFM}$ and the phase shifting method based on six acquisitions

In order to avoid confusion, it is advisable to operate in the field of validity of equation (25) i.e.: $\alpha-\alpha_{c}=0^{\circ}$, or $\alpha-\alpha_{c}= \pm 90^{\circ}$ with $\delta^{c}>\delta$.

\section{Experiments}

The experiments have been performed using a circular polariscope in dark field having quarter wave plates corrected for the wavelength of yellow monochromatic light $\left(\lambda_{0}=589 \mathrm{~nm}\right)$. It has been used:

1. the following light sources:

a. monochromatic sodium vapor lamps that emit at he reference wavelength $\left(\lambda_{0}=589 \mathrm{~nm}\right)$,

b. fluorescent lamps of the energy saving kind (Philips Master 7L-D Super 8018 watt/827) with discrete spectral emission (Fig. 4) having three main narrow band peaks at the following wavelengths: $\lambda_{R}=612 \mathrm{~nm}$ (red), $\lambda_{G}=546 \mathrm{~nm}$ (green), $\lambda_{B}=436 \mathrm{~nm}$ (blue);

2. an RGB camera, model JVC KY-F30 3CCD, with three independent CCD sensors, whose approximate spectral responses are shown in Fig. 5, with a spatial resolution of $768 \times 576$ pixels and with 256 gray levels per color;

3. a polycarbonate (MM PSM1) specimen (Fig. 6) used both as calibration beam and as carrier, and two tempered glass plates (Fig. 7) used for the analysis of residual stresses.

During the acquisition, the magnification has been regulated in such a way that the fringe gradient does not exceed 0.1 orders/pixel [11]. As mentioned above, the calibration procedure, required for the RGB method, was performed using the same specimen (Fig. 6) used as a carrier, in which the maximum fringe orders $\delta=3$ have been produced.

In the case of the RGB method, the search of the retardation was performed using the technique based on the use of a subset of the calibration table [9].

Experimental Results with the Centre Fringe Method in Monochromatic Light

Figure 8 shows the isochromatic fringes in the carrier alone and in the shelf (at the ROI, Fig. 7(a)) superimposed on the carrier. The carrier fringes are deformed due to the residual stresses in the glass. In particular, if the boundary residual stresses in glass are compressive, the resulting fringes (glass superimposed on the carrier) bend toward the compression zone of the carrier (provided that the photoelastic constants of the glass and the carrier have the same
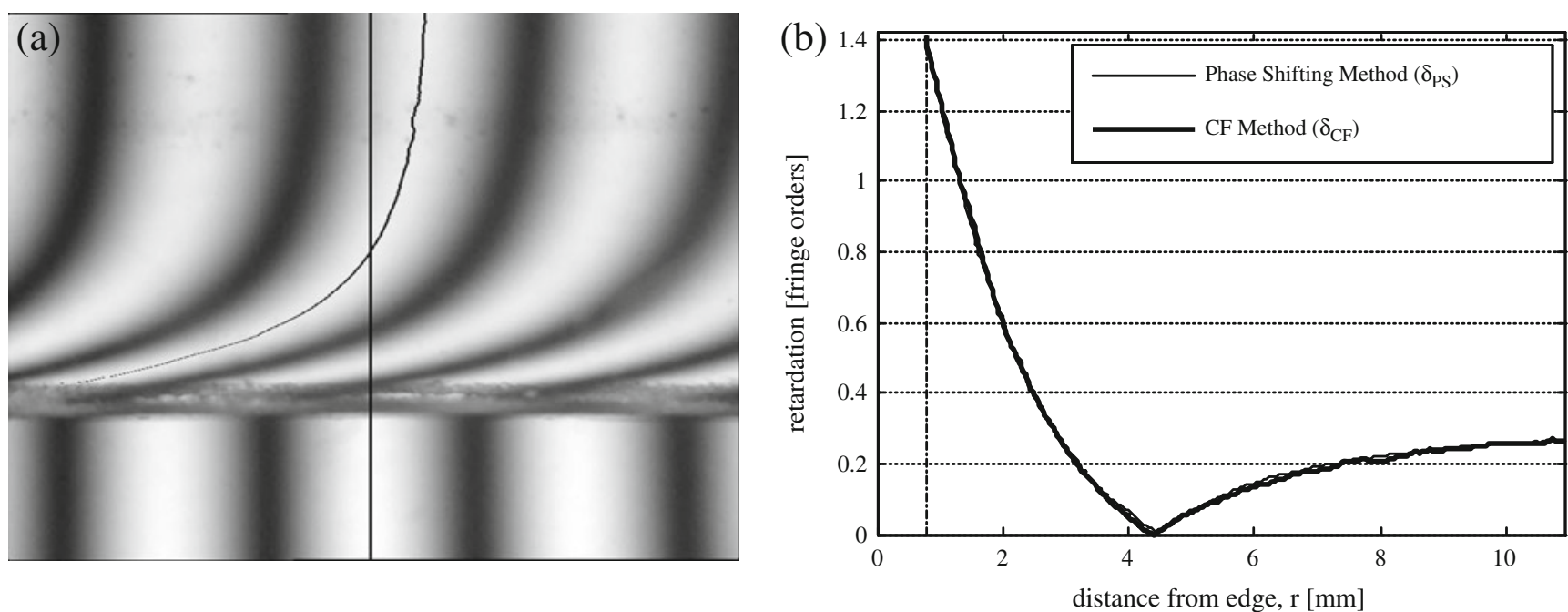

Fig. 11 Car rear window: (a) isochromatic fringes in the ROI (the light fringes used for the CFM method are marked-up), (b) Retardation $\delta$ along the direction $r$ normal to the contour of the glass determined by the CF method 
Fig. 12 White light

isochromatic fringes in dark

field in the carrier alone (up)

and on the shelf superimposed

to the carrier in the shelf (down)

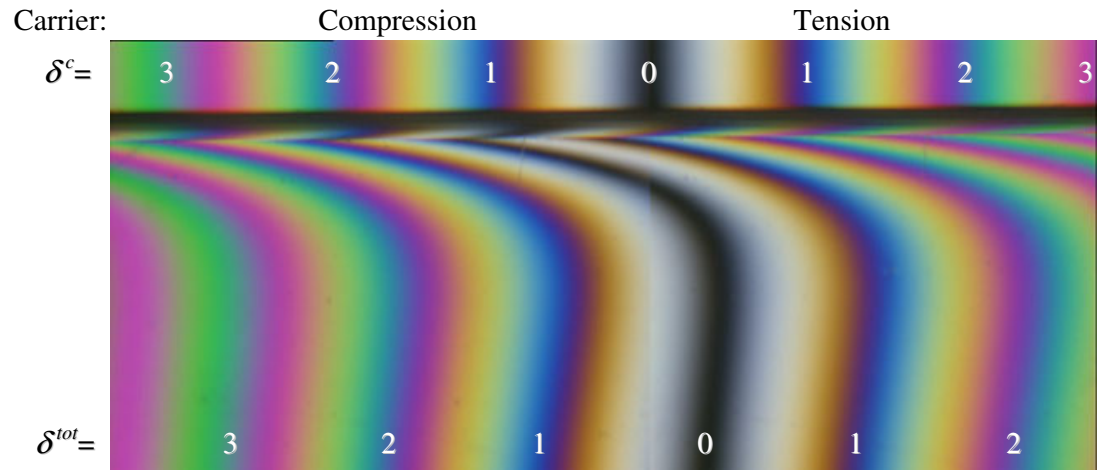

sign). This feature allows the user to identify the sign of the residual stress at the boundary, provided that the sign of the stress in the carrier in known.

Figure 9(a) shows the fringes at the ROI of the shelf. In particular the analysed section is located at the fringe of order 1 in the tensile zone of the carrier. Figure 9(b) shows the retardation along the chosen section as determined by the relationship (16) and by using a six acquisitions phase shifting method [13] for comparison. Almost identical results are obtained by the simplified phase shifting method based on three acquisition [6] and with the phase shifting method in white light [14]. Near the beveled edge of the glass, the retardation is irregular and thus the graph has been truncated near the boundary; in such a case the retardation must be extrapolated at the boundary according to well known procedures as in the case of manual methods $[15,16]$.

Figure 10 shows the errors $e$ with respect to the phase shifting method $\left(e=\delta_{C F}-\delta_{P S}\right)$. These errors are generally lesser than \pm 0.025 fringe orders. Similar results are obtained for the other sections, where the errors lie within the limits mentioned above with some peaks around \pm 0.05 fringe orders.

Figure 11(a) shows the fringes in the ROI of the rear window of the car (see Fig. 7(b)). The analysed section is located at the fringe of order 1.5 in the tensile zone of the carrier. Figure 11(b) shows the retardation along the chosen section as determined by the relationship (16) and the results obtained with the phase shifting method based on six acquisitions [13] for comparison. The errors with
Fig. 13 Shelf: retardation $\delta$ along the direction $r$ normal to the contour of the glass determine by RGB photoelasticity with carrier fringes

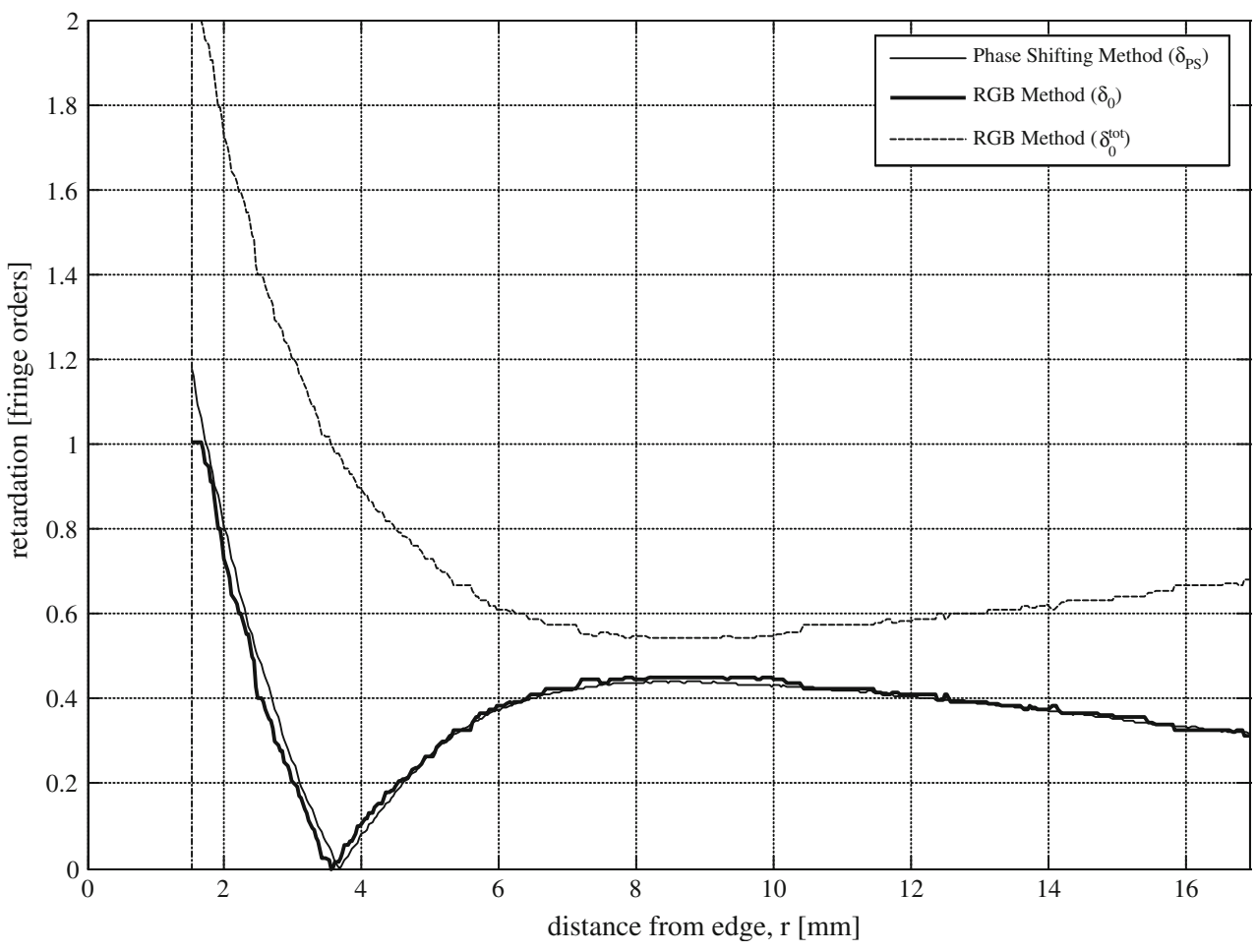




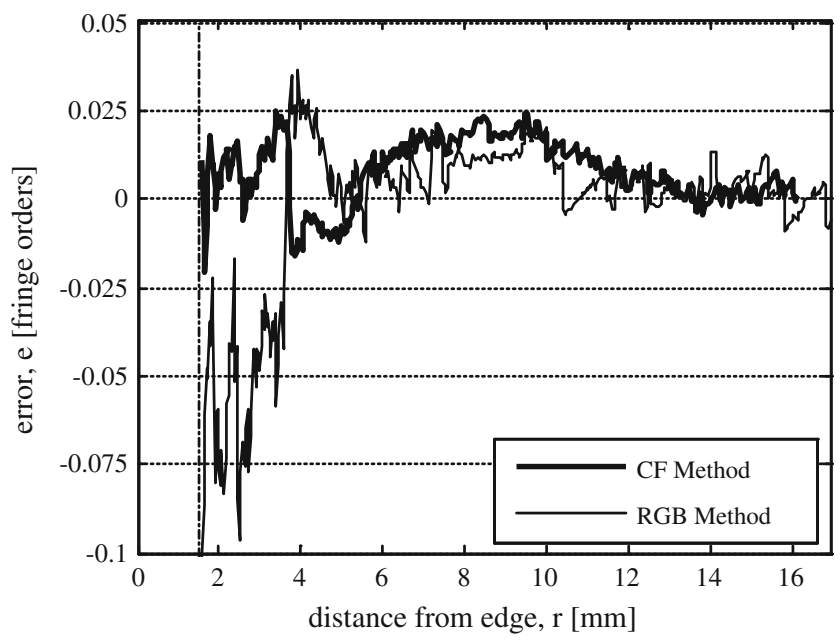

Fig. 14 Shelf: error $e$ between the results obtained by the RGB method $\left(e=\delta_{0}-\delta_{P S}\right)$ and by the CFM $\left(e=\delta_{C F}-\delta_{P S}\right)$ respect to the phase shifting method with six acquisitions

respect to the phase shifting method, $e=\delta_{C F}-\delta_{P S}$, are generally lesser than \pm 0.025 fringe orders, as in the previous case.

Experimental Results in White Light: RGB Photoelasticity with Carrier Fringes

Figure 12 shows the white light isochromatic fringes in the carrier alone and in the shelf superimposed on the carrier at the ROI (Fig. 7(a)).
It was chosen the same measurement section considered for the centre fringe method (located at the fringe of order 1 in the tensile zone of the carrier). In this section the conditions of validity of equation (25) are verified; for this purpose one can simply choose the measuring section (in the glass superimposed on the carrier) in such a way that it is not crossed by the 0 order fringe visible in the glass (Fig. 12); this fringe is easily recognizable because it is the only one appearing black in white light. Figure 13 shows the total retardation $\delta_{0}^{\text {tot }}$ and the retardation $\delta_{0}$ in the glass determined by equation (25) where $\delta_{0}^{c}=1$. For comparison, the figure shows the results obtained by the phase shifting method based on six acquisitions.

Fig. 14 shows the errors with respect to the phase shifting method and, for comparison, the errors obtained by the CFM (also shown in Fig. 10). These errors, except for some isolated peaks, are lesser than \pm 0.05 fringe orders. Similar results are obtained in other sections. The errors of the RGB method, higher than the ones of the CFM, can be due in part to the assumptions made respect to the dispersion of birefringence [see equation (19)] and to the transmittance of the glass [see equations (23)-(24)].

The measurement section of the rear window is the same as that considered for the CFM. In this section the conditions of validity of equation (25) are verified, as in the previous case. Fig. 15 shows the total retardation $\delta_{0}^{\text {tot }}$ and the retardation $\delta_{0}$ in the car rear window determined by equation (25), where $\delta_{0}^{c}=1.5$. For comparison, Fig. 15 shows the results obtained by the phase shifting method
Fig. 15 Rear window of car: retardation $\delta$ along the direction $r$ normal to the contour of the glass determine by RGB photoelasticity with carrier fringes

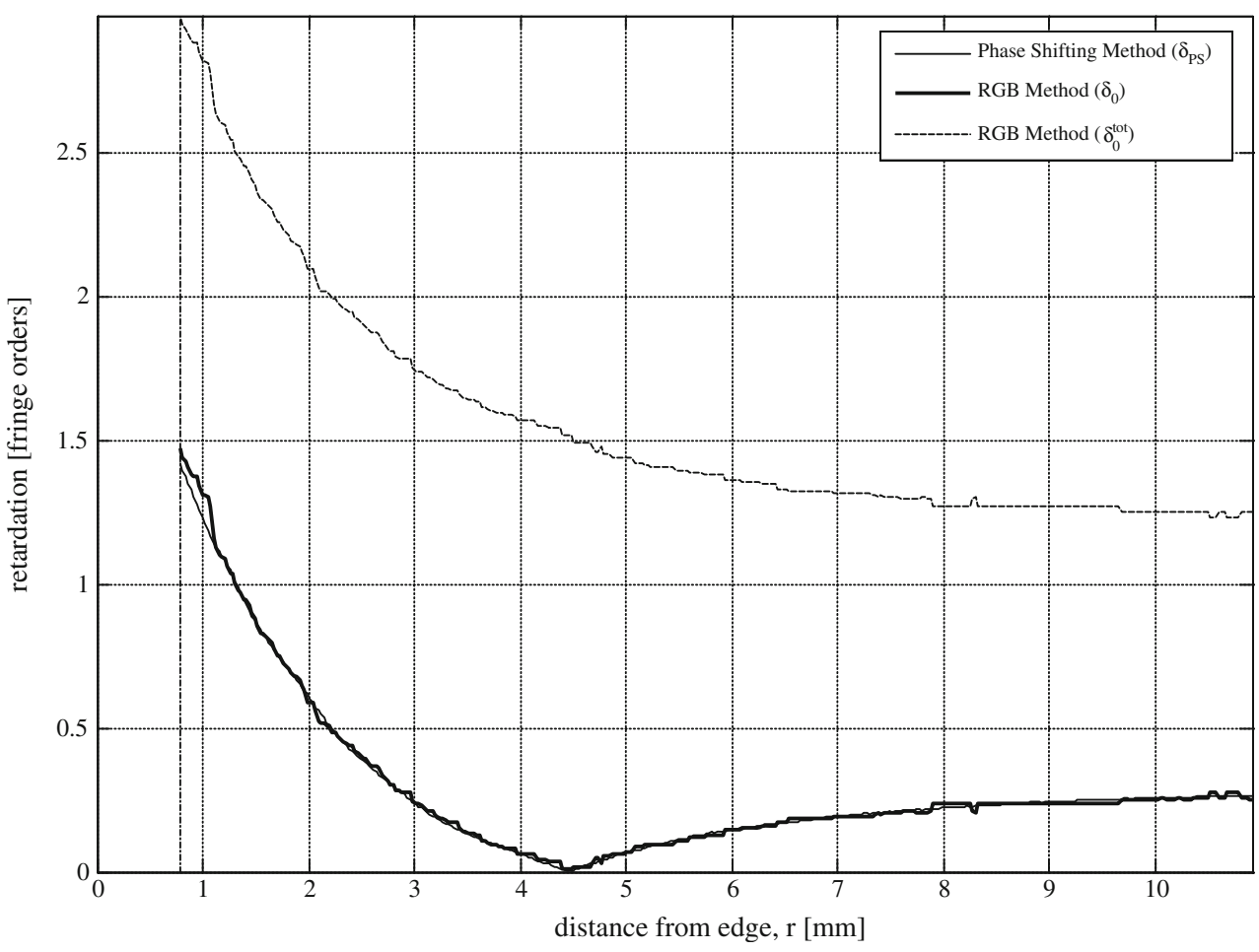


based on six acquisitions. Figure 16 shows the error with respect the phase shifting method and, for comparison, the error obtained by the CFM. These errors, except for some isolated peaks, are lower than \pm 0.02 fringe orders.

\section{Conclusions}

The photoelastic analysis of residual stresses in glass is often based on the use of a Babinet compensator type, or even a simple bent beam, superimposed on the glass specimen. The resulting isochromatic fringes, which are sometimes called test fringes, effectively reveal the presence of residual stresses. This paper proposes the automation of the test fringes method using either the centre fringe method in monochromatic light or RGB photoelasticity in white light.

The quantitative analysis, that does not require additional experimental equipment, shows that the errors (determined by a comparison with results obtained by phase shifting methods) are contained within \pm 0.025 fringe orders for the centre fringe method and within \pm 0.05 fringe orders for RGB photoelasticity. Compared to the phase shifting method (which requires from 3 to 6 acquisitions), the proposed methods have the advantage to require only a single acquisition.

Compared to RGB photoelasticity, the centre fringe method is more easy to apply because it does not require calibration. Conversely, the fringe centre method is limited to straight edges with fringes parallel to the boundaries. In cases in which it can be applied, the classic centre fringe method, now rarely used, in general, proves to be highly effective and easy to use for the analysis of membranal residual stress in glass plates, by using the automated test fringes method described in this paper.

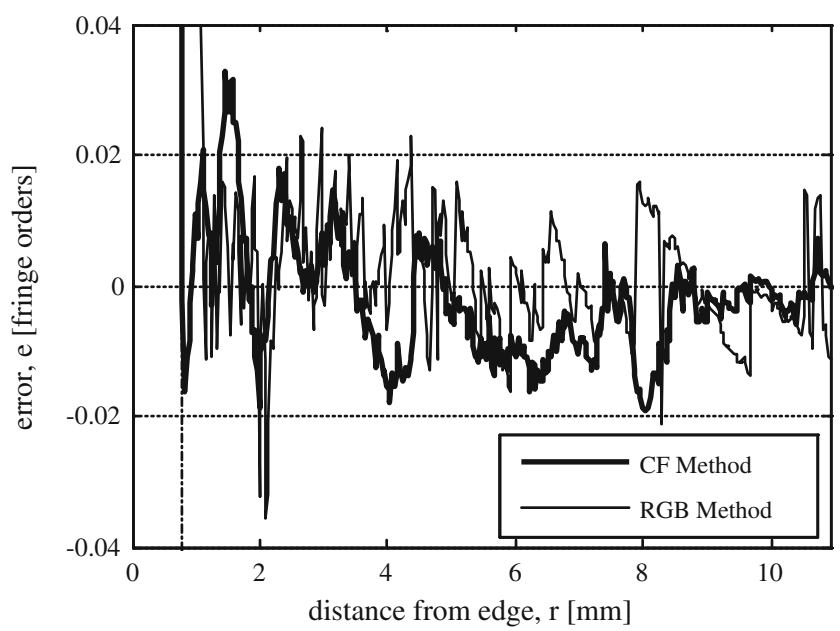

Fig. 16 Car rear window: error $e$ between the results obtained by the RGB method $\left(e=\delta_{0}-\delta_{P S}\right)$ and by the CFM $\left(e=\delta_{\mathrm{CF}}-\delta_{P S}\right)$ respect to the phase shifting method based on six acquisitions
Appendix

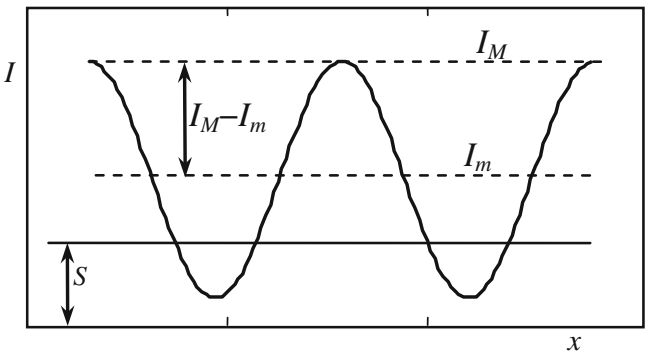

Fig. 17 Determination of the threshold value

\section{Extraction of Fringe Centre}

In this paper the centre fringe extraction has been carried out performing a binarization of the image and applying a thinning algorithm to the binarized image. In particular, for the dark fringes, the following operations are performed:

- determination of average and maximum intensities, $I_{m}$ and $I_{M}$, on the image containing only the carrier (see Fig. 17);

- determination of a threshold value of the dark fringes given by $S=I_{m}-0.65\left(I_{M}-I_{m}\right)$ - see Fig. 17;

- binarization of the image made by letting 1 the intensity of pixels in which $I<S$ (Fig. 18);

- consecutive application of median filtering operations, with circular window increasing from 5 to 13 pixels, in order to eliminate irregularities in the shape of the binarized fringes caused by electronic noise and image defects;

- determination of the positions of the fringes centres by a thinning algorithm, applied to binarized fringes;

- storage of the $x, y$ coordinates of pixels belonging to the fringe under consideration using a simple tracking algorithm.

In the case of the light fringes (corresponding to intermediate fringe orders), the same procedure can be applied, considering a different formulation of the threshold value, i.e. $S^{\prime}=I_{m}+0.65\left(I_{M}-I_{m}\right)$, and letting 1 the intensity of the pixels in which $I>S^{\prime}$.

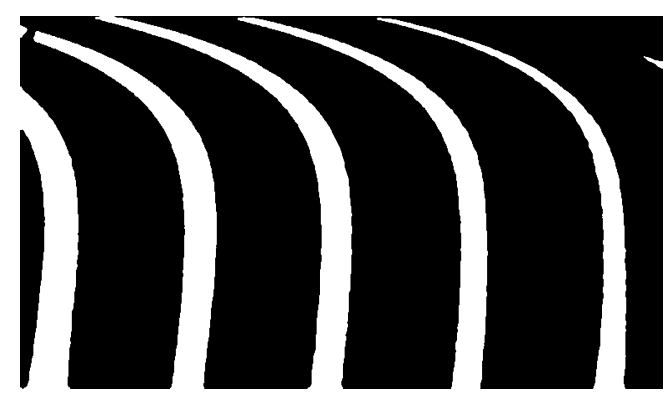

Fig. 18 Binarized isochromatic fringes 
The software was written using the MATLAB programming language. For further details on the method of fringe centre please refer to the copious bibliography on the topic [4].

\section{References}

1. Aben H, Guillemet C (1993) Photoelasticity of glass. Berlin Springer - Verlag

2. McKenzie HW, Hand RJ (1999) Basic optical stress measurement in glass. Society of Glass Technology, Sheffield

3. Aben H, Anton J, Errapart A (2008) Modern photoelasticity for residual stress measurement in glass. Strain 44:40-48

4. Ramesh K (2000) Digital photoelasticity. Springer, Berlin

5. Patterson EA (2002) Digital photoelasticity: principles, practice and potential. Strain 38(1):27-39

6. Ajovalasit A, Petrucci G, Scafidi M (2011) Measurement of edge residual stresses in glass by the phase shifting method. Opt Lasers Eng 49(5):652-657

7. Ajovalasit A, Barone S, Petrucci G (1995) Toward RGB photoelasticity - full field photoelasticity in white light. Exp Mech $35: 193-200$
8. Ramesh K, Deshmukh SS (1996) Three fringe photoelasticityuse of colour image processing hardware to automate ordering of isochromatics. Strain 32:79-86

9. Ajovalasit A, Petrucci G, Scafidi M (2010) RGB photoelasticity: review and improvements. Strain 46:137-147

10. Ajovalasit A, Zuccarello B (2000) Limitation of fourier transform photoelasticity: influence of isoclinics. Exp Mech 40(4):384-392

11. Haake SJ, Patterson EA (1993) The dispersion of birefringence in photoelastic materials. Strain 29(1):3-7

12. Cloud G (1995) Optical methods of engineering analysis. Cambridge University Press

13. Barone S, Burriesci G, Petrucci G (2002) Computer aided photoelasticity by an optimum phase stepping method. Exp Mech 42(2):132-9

14. Ajovalasit A, Petrucci G, Scafidi M (2007) Phase shifting photoelasticity in white light. Opt Lasers Eng 45:596-611

15. Redner AS, Voloshin AS (1990) Surface and face stress in tempered glass. Proc. 9th Int. Conf. on Experimental Mechanics, Copenhagen, vol. 2, 884-891

16. ASTM 1279 , Standard test method for non-destructive photoelastic measurement of edge and surface stresses in annealed, heatstrengthened, and fully tempered flat glass. Annual Book of ASTM standards. 